\title{
Symphonie en Blanc
}

\section{(titre emprunté à}

\section{machin-truc)}

\section{Nicole Dupré}

Elle aimait le blanc; comme on aime les fraises à la crème, les gâteaux au chocolat ou les dimanches pluvieux.

Elle aimait le blanc, sans pour-qui ni pour-quoi; juste comme ça. Tout au plus, quand on 1'accablait de questions se contentait-elle de répéter: "Je n'aime pas les couleurs; le blanc n'est pas une couleur."

Bref, elle aimait le blanc, Monotone? ennuyeux? pas le moins du monde: le blanc offre une palette d'une richesse infinie, subtile en nuances et en dégradés. Le blanc seul permet d'obtenir un véritable camaîeu. Tenez, par exemple: entre un blanc sale (ou blanc douteux) et un blanc Ajax - celui de la Tornade Blanche, pour les poètes de la télé, pour les scientifiques, celui des enzymes gloutons - que de possibilités: il y a le blanc-jaunâtre, un peu passé, ou bien cassé (comme les pois), il y a le blanc phosphorescent, le blanc fluorescent - pas très net, celui-1à::il a beaucoup trop tendance à flirter avec le violet; et il faudra bien un jour se décider à rayer de la liste ce blanc corrompu et perverti. - il y a aussi le blanc crème et le blanc écru, le blanc naturel et le blanc artificiel, le blanc ivoire, le blanc coquille d'oeuf, le blanc papier-mâché, le blanc fleur d'oranger, et des tas d'autres encore, mais ce n'est pas là 1'histoire.

Elle aimait donc le blanc, et par amour du blanc, elle avait choisi de ne s'habiller que de blanc; ce qui est bien naturel. Pourtant, oser affronter le sourire ironique, méprisant, voire sarcastique des vendeuses, à une époque où le pret-à-porter faisait hurler les couleurs et marie les teintes les plus criardes, 
s'opposer aux dictats des couturiers lorsque ces Messieurs de la Haute avaient décidé de "faire chanter les couleurs" "d'êclairer les rues avec des bleus électriques et des verts-pomme, des rouges-dynamite et des jaunes-soleil, des oranges-orange et des violets"... demandait parfois une certaine dose de courage. Fort heureusement, elle avait trouvé en Courrège un allié précieux. Elle avait punaisé au dessus de son 1it, un article découpé dans un magazine, et les jours de cafard noir, elle relisait cet hymne à la blancheur, inventé par le grand mâttre du blanc: "Le BLANC, c'est la couleur du soleil, de la lumière, de la vie. C'est celle du sport - tennis, pelote - des vacances, le signe de la propreté et de la pureté. Mais c'est aussi une couleur qui se marie avec toutes les autres et agrandit les pièces. Elle influence le moral, apporte 1 'apaisement, le recueillement. Elle crée une ambiance sereine, comme dans un couvent: c'est une couleur de paix." ...Wouahou! voilà qui est fort bien dit!

Toujours vêtue de blanc, elle attirait aisément les regards, d'autant plus qu'elle ne se séparait jamais d'une ravissante ombrelle de guipure blanche, découverte aux Puces. L'accessoire était un peu désuet, mais au moins la protégeait des outrages...du soleil. Le soleil! voilà bien une invention démoniaque de ce monsieur Bon-Dieu qu'on dit caché là-hạut dans les nuages! La moindre tache de soleil - je veux dire de rousseur - était une injure à son teint de jeunefille anglaise. Comment imaginer, sur cette perfection, la moindre coloration: "hâle", "bronzage", pire encore, "tanné" étaient pour elle les plus vilains mots de la langue française. Elle avait bien assez de mal à lutter contre cette tendance à rougir au moindre propos un peu vert! Heureusement, un peu de poudre, un peu de fard, rendaient vite à ses joues leur coloration initiale; parfois même, elle trichait un peu, et pour ressembler un peu plus à $1^{\prime}$ Ophélie du 


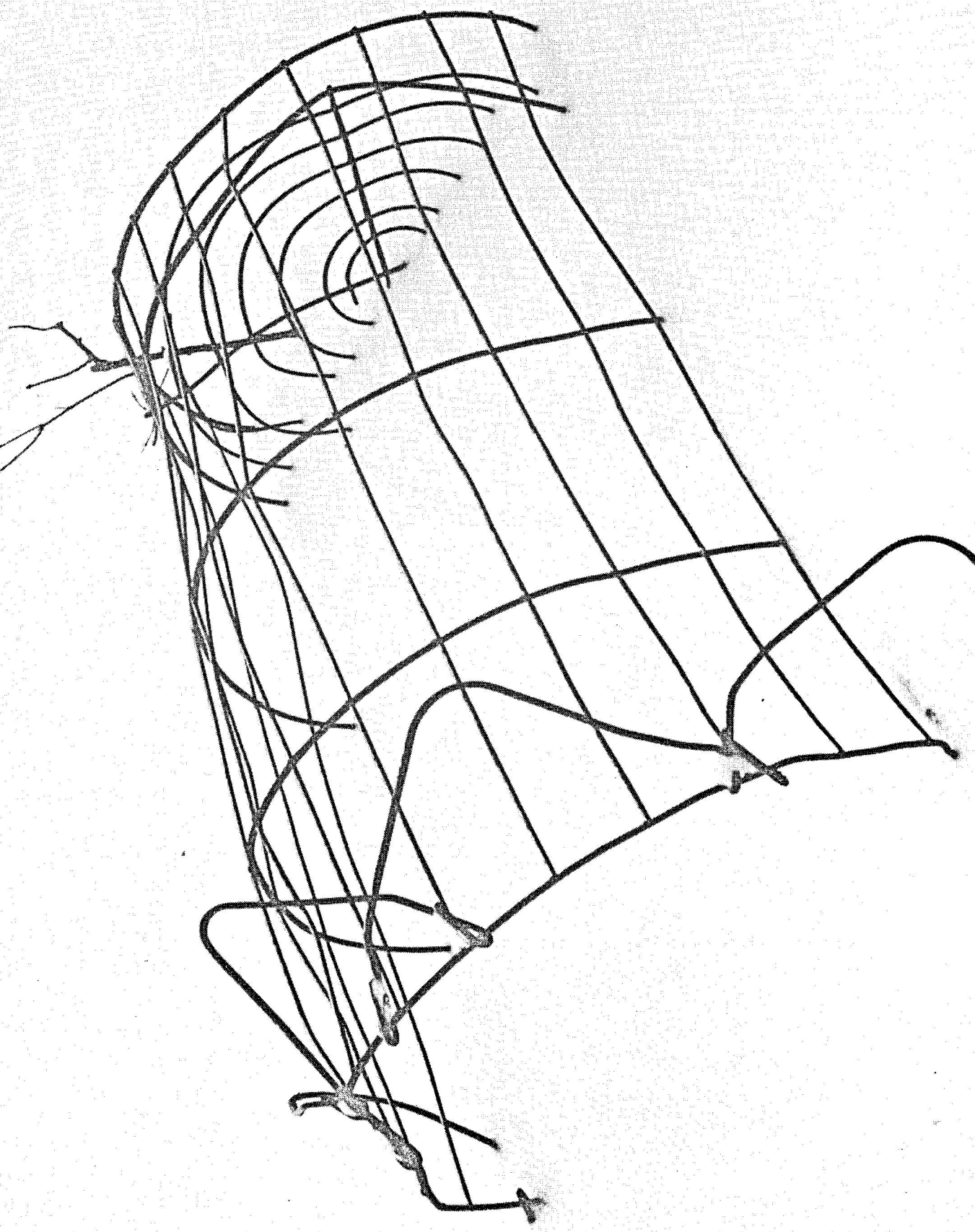


Rimbaud;

"La blanche Ophélie flottait comme un grand lys $(\ldots)$

o pâle Ophélie! belle comme la neige!" elle corrigeait, grâce à l'art savant du maquillage, les imperfections de Mère Nature. Le plus difficile était d'atténuer 1 'éclat de sa bouche purpurine: après tout, un blanc-à-lèvres, ce n'est pas monnaie courante... Quelquefois, bien sûr, ses efforts lui valaient quelques remarques désobligeantes, du genre: "Vise un peu la gueule enfarinée..."; mais c'étaient là propos de peu de poids lorsque son miroir, le confident de ses peines, l'ami fidèle qui ne trahit jamais, confirmait sa ressemblance avec Bip, 1'adorable créature du mime Marceau, au visage si blanc... $N^{\prime}$ 'allez pas croire, avrès tout cela, qu'il s'agissait d'une lubie, d'ine manie, d'une folie. Non, pas du tout: c'était beaucoup plus sérieux que cela et son amour du blanc était à la fois une éthique et une esthétique: parfaitement! ou si vous préférez, une philosophie du Bien et du Beau (avec des tas de B majuscules), une morale du raffinement, une exigence... Choisir le blanc, c'était choisir une façon de s'habiller, mais aussi une façon de bouger, une façon d'habiter, une façon de penser, de VIVRE, d'ETRE......

LE BLANC COMME UNE DIETETIQUE: E11e, (nous 1'appellerons Blanche, si vous voulez bien; si vous ne voulez pas... nous 1'appellerons Blanche, aússi.) elle donc, sélectionne avec soin sa nourriture, et préfère les viandes blanches au viandes rouges (cela va de soi) et le blanc de poulet à toute autre volaille. Pour l'épicerie, pas beaucoup de problèmes: sucre, sel, riz, farine, la farine surtout, si blanche, si pure... le choix est plus restreint quant aux légumes, mais les choux-fleurs et les champignons de Paris s'épanouissent presque tout au long de l'année; et puis, elle s'en'tire toujours, tant bien que mal, en faisant blanchir ses coeurs d'artichauts comme le 
recommandent la plupart des recettes de cuisine, ou en préparant des plats en sauce blanche ( il faut avouer qu'elle a un petit faible pour la béchamel, et une petite tendance à en user et abuser: pensez: un beurre blanc, un peu de farine, une pincée de sel et du lait, si blanc, si doux, voilà de quoi combler tous ses rêves culinaires! Ceci dit, si le plat de résistance laisse un peu à désirer, elle peut toujours se rattraper sur les fromages; Là, elle laissera volontiers de côté les bleus d'Auvergne et autres Roquefort pour se tourner vers ses préférés: un bon fromage de chèvre, attendrissant à force de mousse, ou le candide fromage blanc, un peu fade peut-être, mais le seul qui ne $l^{\prime}$ ait jamais trahit. Quant aux desserts... ils s'offrent à foison et rivalisent en dêbauche... de blancheur: oeufs en neige, crème Chantilly, meringue, ou mieux encore, ces merveilleuses sucreries qui empruntent aux splendeurs enneigées leurs patronymes: Mont-Blanc, Himalaya, que d'extases vous suggérez! ... Pour étancher sa soif, elle a renoncé une fois pour tout aux vins rouges, décidément trop vulgaires, en faveur des vins blancs (non sans regrets peut-être, mais blancheur oblige).

LE BLANC COMME UNE ESTHETIQUE: Son appartement bien sûr, est tout en blancheur confondue: les murs, les plafonds, les meubles, les tissus, tout est blanc, Mais son salon est sa grande fierté: rien de plus facile que de repeindre en blanc un vieux parquet ciré, de le réchauffer d'un tapis de haute laine naturelle et d'une peau de chèvre, aux longs poils soyeux... et blancs; quelques coussins bourrus, une télévision ronde en plastique blanc, modèle Philips 1970, publicité non payée; de toute façon elle ne regarde jamais que les ëmissions avec carré blanc; des disques? pas question, tant que les maisons d'édition ne se décideront pas à produire autre chose 
que ces horribles ronds de je-ne-sais-quoi noir! Quelques fleurs pour égayer l'ensemble; mais seules les marguerites ont droit d'entrée au Io de la rue Blanche; les roses blanches sont vraiment devenues trop banales depuis la chanson, comme les pommiers blancs et les cerisiers roses: pouah! à la rigueur, les lys, pour leurs senteurs parfumées, ou mieux une orchidée rare; mais elles sont vraiment devenues trop rares ces derniers temps; les fleurs d'accacias aussi ont sa faveur, celles qu'on attrape en sautant après les branches les plus basses, celles pour lesquelles on s'écorche un peu le bout des doigts, mais qu'on machouille pendant des heures et qui laissent un goût sucré sur les lèvres. Aux murs, quelques toiles blanches. Rien de plus insupportable que ces peintres maladroits et sans goût qui, à coups de brosses et de pinceaux, de couteaux et de pistolets, assassinent la blancheur virginale d'une toile.

Sa bibliothèque lui a donné bien du mal: elle s'est d'abord contentée de recouvrir chaque volume d'une jolie liseuse d'un blanc immaculé; ensuite elle s'est bien gardée d'ouvrir jamais un seul de ces affreux bouquins car la vue de tous ces kilos de papier noircis par l'encre d'imprimerie lui soulevait le coeur; bientôt, 1 'idée même de toutes ces petites lettres souillant la page blanche lui est devenue insupportable et dans un grand élan idéaliste, mystique, elle s'est débarrassée en deux temps, trois mouvements et quatre allumettes, de tous ces objets importuns. Un livre, un seul a échappé à la débacle, un livre unique, et rare, un livre comme une pierre précieuse, comme le premier homme sur la lune, comme un rayon de soleil en plein décembre, un livre enfin dont toutes les pages sont blanches, le seul dont elle ne se lasse jamais, le seul qui se renouvelle à chaque lecture. Quelques exceptions quand même: à côté de ce livre des merveilles, elle a conservé quelques poèmes, soigneusement recopiés à l'encre sympathique: un poème de Mallarmé; pour un vers qui constitue à lui seul un art d'écrire... ou de ne pas 
écrire:

"Et le vide papier que 1a blancheur défend" La Chanson du Mal-Aimé d'Apollinaire a elle aussi échappé au massacre, pour ses rêves de blancheur au rythme obsessif:

"Voie lactée ô soeur lumineuse

Des blancs ruisseaux de Chanaan

Et des corps blancs des amoureuses"

En poésie, au moins, la valeur du blanc est reconnue; on ne peut pas en dire autant des romans; de tout ce fratras de paroles inutiles, elle ne put guère sauver que Moby Dick, la jolie baleine blanche aux yeux bleus, et, mais avec beaucoup de condescendance, Marguerite Gauthier, pour ses camélias blancs - et encore, pas tous les jours...

Un moment intriguée par Théophile Gautier, elle a essayé de mettre en musique sa Symphonie en Blanc Majeur - un magnifique Pleyel, tout relaqué de blanc par ses propres soins, fait 1 'honneur de son salon mais comme elle se refusait à jouer autre chose que des blanches, le rythme était un peu lent, et monotone, et lourd. Et puis, sans dièse ni bémo1, la mélodie aussi était un peu monocorde. I1 faut avouer que ces touches noires sur l'ivoire du clavier l'ont longtemps obsédée, jusqu'au jour où elle eut l'idée géniale de les peindre en blanc, tout simplement.

Le cinéma? elle y a renoncé depuis longtemps. dans les années cinquante et soixante, on pouvait encore espérer voir un bon film en noir et blanc; mais maintenant, les producteurs se sont tous entichés de couleurs... tant pis pour eux! Et puis d'ailleurs, les salles obscures sont vraiment trop obscures...

BLANCHE, puisque tel est bien son nom, est une jeune fille pleine de talents et de promesses. Intelligente, elle brille surtout dans les salons, où elle $n^{\prime}$ a pas son pareil pour mettre en valeur les blancs de la conversation. Elle interrompit ses études le 
jour où un professeur un peu vicieux - en fait, ils sont tous un peu zin-zin dans le métier - proposa à ses étudiants, deux sujets au choix (ce qui peut passer pour une preuve de magnanimité mais constitue en réalité un supplice raffiné): une explication du sonnet des Voyelles de Rimbaud, ou une étude de la symbolique des couleurs dans Phèdre (de Racine, bien sûr, comme si tout le monde ne le savait pas!) noir-crime, rouge-sang, jaune-soleil d'un côté, l'arc-en-ciel de l'autre côté! cruel dilemme, comble d'horreur. Elle s'est, faut-il en douter, empressée de rendre feuille-blanche, abandonnant ainsi sa brillante carrière universitaire (selon l'expression consacrée) sur un coup de tête. . . et un point d'interrogation! Il y a des choses comme ça qu'un être humain ne peut pas supporter, ne doit pas accepter. Ainsi, libérée de ce carcan social, elle dispose maintenant du plus clair de son temps et passe ses nuits blanches à rêver d'un pays où tout serait blanc, blanc, blanc, blanc, blanc, blanc, blanc, blanc, blanc,

."Un pays tropical, avec des plages de sable blanc, un pays où tout serait blanc à force de lumière; un ciel blanc de chaleur" dit Camus en parlant de Tipasa. . . ça doit être chouette un ciel tout blanc, au lieu de ce bleu bête et stupide . . oui, mais un pays tropical ça veut dire aussi des marchés pittoresques et l'agression colorée des mangues et des papayes. Quelle horreur! Sans compter les couleurs de l'exotisme et les costumes brodés, tissés dans les coloris les plus chatoyants: l'éclatement des couleurs: pouah!

- . Alors, un pays blanc de neige et de glaces, un pays d'ours blancs et de renards blancs (voilà des animaux bien sympathiques!) un pays où lorsque souffle le vent, le ciel et la terre sont en blancheur confondus, un pays. .

. . Un pays où je retrouverais mes personnages favoris, Blanche-Neige et Blanche de Castille, 
Henry IV, à cause de son panache blanc et Galaad, le blanc chevalier. . ."

Blanche rêve, Blanche sourit, Blanche soupire

Arrive le jour fatal, sombre parmi les sombres, un jour à marquer d'une pierre noire, un jour de deuil et de tristesse où Blanche commet 1'irréparable, L A F A U T : elle aime. .

Bien sûr, ce n'est pas la première fois. I1 lui est dêjà arrivê d'être amoureuse; mais pour quelques secondes seulement, quand le monsieur, sur le chapeau duquel elle était assise, s'était levé, blanc de rage: quel émoi! mais comme le dit monsieur, dans une colère bleue, tournait au rouge, elle avait jugé qu'après tout, ça ne valait pas la peine.

Bref, elle aime pour de bon, cette fois, elle aime d'un amour fugace et fugitif, qui ne laisse au coeur que des regrets, et dans son sein, la fleur fragile et tendre, fraîchement épanouie, je veux dire les prémices de 1 'enfant que pendant neuf mois elle attendra.

En fait, elle se soucie fort peu du temps qui passe: neuf mois ou neuf jours, quelle importance? quelle est la différence? Pendant neuf mois (ou neuf jours), elle s'épanouit dans des montagnes de laines soyeuses et angora, elle palpite comme un oiseau blessé (un cliché de plus à ne pas rater) parmi des kilomètres de rubans, de dentelles, d'organdie, de plumetis, de soie et de linon, pour achever le plus beau de ses rêves: une layette immaculée!

L'hôpital ensuite; moins désagréable qu'on ne pourrait le croire; là aussi le blanc triomphe, dans les chambres et les couloirs, sur les blouses des médecins et celles des infirmières. 
Puis, un grand silence blanc.

Atmosphère ouatée; tête cotonneuse, elle se réveille enfin, et ouvre les yeux sur le plus adorable, le plus attendrissant, le plus émouvant, le plus surprenant. . . (cf. Madame de Sévigné) des bébés: un petit garçon resplendissant de santé, éclatant de vie, un petit garçon parfait, un petit garçon. - NOIR; ni métis ni mulâtre, ni café-au-lait ni olivâtre, mais d'un noir profond, indélébile, un noir d'ébène (ou noir chocolat), un noir absolu, un noir parfait.

Stupeur et stupêfaction, catastrophe et cataclysme, désespoir et damnation, calembour et calamité, patati et patata: tant de blancheur pour arriver à ça! Patata et patati, calamité et calembour, damnation et désespoir, cataclysme et catastrophe, stupéfaction et stupeur.

Une seule solution: le suicide! Mais comment? se noyer? non, les cadavres des noyés sont gonflés et verts; s'asphyxier au gaz? non, les cadavres sont trop laids: rose dragée, beurk! se couper les poignets? avec du sang partout? ah non, ça n'est vraiment pas propre et de toute façon, le rouge, c'est pas beau; s'empoisonner aux barbituriques? le mot est bien joli, mais ça ne marche jamais et on en voit de toutes les couleurs.

Alors, tout bêtement, tout simplement, et pour en finir une bonne fois, elle cesse de respirer, comme ça, sans plus d'histoire.

The University of Kansas 